OPEN ACCESS

Edited by:

Weihui Wu.

Nankai University, China

Reviewed by:

Krisztina M. Papp-Wallace, Louis Stokes Cleveland VA Medical

Center, United States

Marisa Winkler,

Brigham and Women's Hospital and Harvard Medical School,

United States

*Correspondence:

Esther Recacha

erecachavillamor@gmail.com

Specialty section

This article was submitted to Antimicrobials, Resistance

and Chemotherapy,

a section of the journal

Frontiers in Microbiology

Received: 14 January 2021

Accepted: 15 March 2021

Published: 07 April 2021

Citation:

Recacha E, Fox V, Díaz-Díaz S,

García-Duque A, Docobo-Pérez F, Pascual Á and Rodríguez-Martínez JM (2021) Disbalancing Envelope Stress Responses as a Strategy for Sensitization of Escherichia coll to Antimicrobial Agents.

Front. Microbiol. 12:653479. doi: 10.3389/fmicb.2021.653479

\section{Disbalancing Envelope Stress Responses as a Strategy for Sensitization of Escherichia coli to Antimicrobial Agents}

\author{
Esther Recacha1,2,3*, Valeria Fox ${ }^{4}$, Sara Díaz-Díaz ${ }^{2,3,5}$, Ana García-Duque1, \\ Fernando Docobo-Pérez ${ }^{2,3,5}$, Álvaro Pascuall,2,3,5 and \\ José Manuel Rodríguez-Martínez ${ }^{2,3,5}$
}

1 Unidad Clínica de Enfermedades Infecciosas, Microbiología y Medicina Preventiva, Hospital Universitario Virgen Macarena, Seville, Spain, ${ }^{2}$ Red Española de Investigación en Patología Infecciosa (REIPI), Instituto de Salud Carlos III, Madrid, Spain, ${ }^{3}$ Instituto de Biomedicina de Sevilla (IBiS), Hospital Universitario Virgen del Rocío/CSIC/Universidad de Sevilla, Seville, Spain, ${ }^{4}$ Laboratory of Molecular Microbiology and Biotechnology, Department of Medical Biotechnologies, University of Siena, Siena, Italy, ${ }^{5}$ Departamento de Microbiología, Universidad de Sevilla, Seville, Spain

Disbalancing envelope stress responses was investigated as a strategy for sensitization of Escherichia coli to antimicrobial agents. Seventeen isogenic strains were selected from the KEIO collection with deletions in genes corresponding to the $\sigma^{\mathrm{E}}$, Cpx, Rcs, Bae, and Psp responses. Antimicrobial activity against 20 drugs with different targets was evaluated by disk diffusion and gradient strip tests. Growth curves and timekill curves were also determined for selected mutant-antimicrobial combinations. An increase in susceptibility to ampicillin, ceftazidime, cefepime, aztreonam, ertapenem, and fosfomycin was detected. Growth curves for Psp response mutants showed a decrease in optical density (OD) using sub-MIC concentrations of ceftazidime and aztreonam ( $\triangle p s p A$ and $\triangle p s p B$ mutants), cefepime ( $\triangle p s p B$ and $\triangle p s p C$ mutants) and ertapenem ( $\triangle p s p B$ mutant). Time-kill curves were also performed using $1 \times \mathrm{XMIC}$ concentrations of these antimicrobials. For ceftazidime, $2.9 \log _{10}(\triangle p s p A$ mutant) and $0.9 \log _{10}(\triangle p s p B$ mutant) decreases were observed at 24 and $8 \mathrm{~h}$, respectively. For aztreonam, a decrease of $3.1 \log _{10}\left(\Delta p s p A\right.$ mutant) and $4 \log _{10} 0_{10}(\Delta p s p B$ mutant) was shown after $4-6 \mathrm{~h}$. For cefepime, $4.2 \log _{10}\left(\Delta p s p B\right.$ mutant) and $2.6 \log _{10}(\Delta p s p C$ mutant) decreases were observed at 8 and $4 \mathrm{~h}$, respectively. For ertapenem, a decrease of up to $6 \log _{10}(\Delta p s p B$ mutant) was observed at $24 \mathrm{~h}$. A deficient Psp envelope stress response increased $E$. coli susceptibility to beta-lactam agents such as cefepime, ceftazidime, aztreonam and ertapenem. Its role in repairing extensive inner membrane disruptions makes this pathway essential to bacterial survival, so that disbalancing the Psp response could be an appropriate target for sensitization strategies.

Keywords: envelope stress responses, bacterial sensitization, beta-lactams, antimicrobial resistance, Gramnegative bacteria 


\section{INTRODUCTION}

Since antimicrobial resistance is increasing worldwide, new targets (Dickey et al., 2017; Recacha et al., 2017; Cattoir and Felden, 2019) need to be sought, either to find new antimicrobial families or to increase the susceptibility of bacterial populations (Laxminarayan et al., 2013). Envelope stress responses are important pathways for bacterial survival in the presence of stressors, including antimicrobials (Guest and Raivio, 2016; Hersch et al., 2020), and their alteration could be proposed as a strategy for weakening bacteria. The Gram-negative envelope is composed of inner membrane (IM), periplasm, containing a thin peptidoglycan (PG) layer, and outer membrane (OM). This envelope provides Gram-negative bacteria with protection against external environmental agents, including antibiotics (Silhavy et al., 2010). The $\sigma^{\mathrm{E}}, \mathrm{Cpx}, \mathrm{Rcs}$, Bae and Psp systems are the main envelope stress response pathways in Gram-negative bacteria for restoring homeostasis to cells with induced envelope damage and are activated in different ways (Guest and Raivio, 2016; Mitchell and Silhavy, 2019). The $\sigma^{\mathrm{E}}$ response detects perturbations in outer membrane (OM) or lipopolysaccharide (LPS) biogenesis through interactions between either the exposed C-terminus of misfolded outer membrane proteins (OMPs) and the DegS periplasmic protease, or between the anti-anti-s factor RseB and periplasmic LPS molecules, respectively. These both initiate a regulated intramembrane proteolysis cascade ultimately leading to the liberation of $\sigma^{\mathrm{E}}$ from a membranebound anti-sigma factor and the upregulation of adaptive factors, including chaperones, proteases, membrane biogenesis proteins, and a set of small RNAs that downregulate OMP production (Ades, 2004; Ruiz and Silhavy, 2005; ValentinHansen et al., 2007; Lima et al., 2013; Flores-Kim and Darwin, 2014; Kim, 2015). The Cpx response is regulated by the CpxA sensor kinase and response regulator CpxR. Envelope stresses causing protein misfolding, and adhesion, inactivate the inhibitor CpxP, trigger CpxA-mediated phosphorylation of CpxR, and altered expression of protein foldases and proteases, respiratory complexes, transporters, and cell wall biogenesis enzymes that impact resistance to a number of antibiotics, particularly aminoglycosides (Raivio, 2014). The Rcs response is regulated by a two-component phosphorelay consisting of two inner membrane (IM)-associated sensor kinase molecules, $\mathrm{RcsC}$ and $\mathrm{RcsD}$, together with a cytoplasmic response regulator, RcsB. Multiple environmental parameters and conditions leading to a weakened envelope activate $\mathrm{RcsC}$ and/or RcsD, which together catalyze the phosphorylation of RcsB, leading to changes in the expression of genes associated with capsule production, motility, virulence, biofilm formation, and other envelope proteins (Majdalani and Gottesman, 2005; Huang et al., 2006). The Rcs pathway has been linked to resistance to a number of microbially and host produced antimicrobials including beta-lactam antibiotics, cationic antimicrobial peptides and bile (Hirakawa et al., 2003; Erickson and Detweiler, 2006; Laubacher and Ades, 2008; Farris et al., 2010; Farizano et al., 2014). The Psp response is activated by changes linked to the aberrant localization of OM secretin complexes and other conditions that disrupt the IM, including the dissipation of the proton motive force. These signals are transduced through changes in interactions between a set of Psp proteins that ultimately lead to the liberation of the PspF transcription factor from the inhibitor PspA and the upregulated production of a limited set of adaptive factors capable of fostering endurance and survival (Flores-Kim and Darwin, 2014, 2016). Finally, Bae response is controlled by the two-component system made up of the sensor kinase BaeS and its cognate partner BaeR. This pathway is activated by antimicrobial compounds made by plants, animals, and microbes, as well as metals, and can stimulate resistance to broad classes of these substances, primarily, it appears, through the regulation of the multidrug RND efflux pumps AcrD and MdtABC, together with the common OM component TolC (Baranova and Nikaido, 2002; Raffa and Raivio, 2002; Cordeiro et al., 2014; Lin et al., 2014).

The aim of this study was to investigate the effect of alteration of the envelope stress response pathways of the $\sigma^{E}$, Cpx, Rcs, Bae, and Psp systems on sensitization to antimicrobial agents targeting the bacterial cell wall, protein, RNA, DNA or folic acid synthesis.

\section{MATERIALS AND METHODS}

\section{Bacterial Strains}

A set of 17 E. coli strains derived from E. coli BW25113 belonging to the KEIO collection were used (Baba et al., 2006). Strains were selected with defective envelope stress responses, with deletions in genes for the $\sigma^{\mathrm{E}}$ (rseA and $r s e B$ genes), Cpx (cpxA, cpxR, cpxP, and $n l p E$ genes), Rcs ( $r c s F, r c s A, r c s C, r c s D$, and $r c s B$ genes), Bae (baeR and baeS genes) and Psp responses ( $p s p A, p s p B, p s p C$, and $p s p F$ genes) (Supplementary Table 1). Each deletion was verified by PCR (Supplementary Table 2 ).

\section{Antimicrobial Susceptibility Testing}

Antimicrobial susceptibility was determined by disk diffusion (Oxoid ${ }^{\circledR}$, United Kingdom) and gradient strip tests (Liofilchem ${ }^{\circledR}$, Italy), using CLSI reference methods (Clinical and Laboratory Standards Institute, 2016.). Any mutant-antimicrobial combination with a halo size that differed by more than $3 \mathrm{~mm}$ by disk diffusion from the wild-type (E. coli BW25113) was selected for the gradient strip test.

The antimicrobials used were: penicillin G, ampicillin, amoxicillin/clavulanic acid, cefoxitin, ceftazidime, cefepime, ertapenem, imipenem, aztreonam, gentamicin, amikacin, tetracycline, chloramphenicol, colistin, rifampicin, nalidixic acid, ciprofloxacin, sulfonamides compound, sulfamethoxazole/ trimethoprim, and fosfomycin.

\section{Growth Curve Assays}

Growth curves were performed for mutant-antimicrobial combinations with a decrease of MIC determined by gradient strip tests. Psp mutants (except $\Delta p s p F$ ) were tested to betalactams agents listed in Table 1 despite not showing decreases in MIC value. After overnight culture in Mueller-Hinton broth (MHB) at $37^{\circ} \mathrm{C}$, bacterial suspensions were diluted to achieve an $\mathrm{OD}_{625 \mathrm{~nm}}$ of 0.1 ( $\left.\mathrm{ca} .10^{8} \mathrm{CFU} / \mathrm{mL}\right)$, then diluted $10^{-4}$-fold in $\mathrm{MHB}$ medium containing sublethal concentrations $(0.5 \mathrm{xMIC}$ and 
TABLE 1 | Susceptibility test determined by gradient strip tests.

\begin{tabular}{|c|c|c|c|c|c|c|c|c|c|c|c|c|c|c|c|c|c|c|c|}
\hline & Strain & $\begin{array}{l}\text { MIC }^{a} \\
\text { AMP }\end{array}$ & $\begin{array}{c}\text { Fold } \\
\text { change }^{b}\end{array}$ & $\begin{array}{l}\text { MIC } \\
\text { CAZ }\end{array}$ & $\begin{array}{l}\text { Fold } \\
\text { change }\end{array}$ & $\begin{array}{l}\text { MIC } \\
\text { FEP }\end{array}$ & $\begin{array}{l}\text { Fold } \\
\text { change }\end{array}$ & $\begin{array}{l}\text { MIC } \\
\text { ETP }\end{array}$ & $\begin{array}{c}\text { Fold } \\
\text { change }\end{array}$ & $\begin{array}{l}\text { MIC } \\
\text { ATM }\end{array}$ & $\begin{array}{l}\text { Fold } \\
\text { change }\end{array}$ & $\begin{array}{l}\text { MIC } \\
\text { FOS }\end{array}$ & $\begin{array}{c}\text { Fold } \\
\text { change }\end{array}$ & $\begin{array}{l}\text { MIC } \\
\text { IPM }\end{array}$ & $\begin{array}{c}\text { Fold } \\
\text { change }\end{array}$ & $\begin{array}{l}\text { MIC } \\
\text { AK }\end{array}$ & $\begin{array}{c}\text { Fold } \\
\text { change }\end{array}$ & $\begin{array}{c}\text { MIC } \\
\text { C }\end{array}$ & $\begin{array}{c}\text { Fold } \\
\text { change }\end{array}$ \\
\hline & $\mathrm{BW}^{\mathrm{C}}$ & 6 & & 0.19 & & 0.032 & & 0.012 & & 0.047 & & 0.5 & & 0.19 & & 0.5 & & 8 & \\
\hline$\sigma^{\mathrm{E}}$ response & $\Delta r s e A$ & - & & - & & - & & - & & - & & 0.5 & 1 & - & & - & & - & \\
\hline \multirow[t]{3}{*}{ Cpx response } & $\Delta c p \times A$ & - & & - & & - & & 0.016 & 0.75 & - & & - & & - & & - & & 8 & 1 \\
\hline & $\Delta c p \times R$ & 8 & 0.75 & 0.19 & 1 & 0.032 & 1 & 0.012 & 1 & 0.047 & 1 & 0.5 & 1 & - & & 2 & 0.25 & - & \\
\hline & $\Delta c p x P$ & - & & - & & - & & - & & 0.047 & 1 & 0.5 & 1 & - & & - & & - & \\
\hline \multirow[t]{4}{*}{ Rcs response } & $\Delta r c s F$ & - & & - & & - & & 0.016 & 0.75 & - & & - & & 0.19 & 1 & - & & - & \\
\hline & $\Delta r c s C$ & - & & - & & 0.064 & 0.5 & 0.016 & 0.75 & - & & 0.5 & 1 & 0.25 & 0.76 & - & & - & \\
\hline & $\Delta r c s D$ & - & & 0.19 & 1 & 0.032 & 1 & 0.012 & 1 & 0.032 & 1.5 & 0.38 & 1.3 & - & & - & & - & \\
\hline & $\Delta r c s B$ & - & & 0.19 & 1 & 0.032 & 1 & 0.012 & 1 & 0.047 & 1 & 0.5 & 1 & 0.19 & 1 & - & & - & \\
\hline \multirow[t]{2}{*}{ Bae response } & $\Delta$ bae $R$ & - & & 0.125 & 1 & 0.032 & 1 & 0.012 & 1 & 0.032 & 1.5 & 0.5 & 1 & - & & - & & 8 & 1 \\
\hline & $\Delta$ baes & & & - & & - & & - & & - & & 0.5 & 1 & - & & - & & - & \\
\hline \multirow[t]{4}{*}{ Psp response } & $\Delta p s p A$ & - & & 0.094 & 2 & 0.023 & 1.4 & 0.008 & 1.5 & 0.047 & 1 & - & & - & & - & & - & \\
\hline & $\Delta p s p B$ & - & & 0.094 & 2 & 0.023 & 1.4 & 0.012 & 1 & - & & - & & - & & - & & - & \\
\hline & $\Delta p s p C$ & 4 & 1.5 & 0.125 & 1.5 & - & & 0.012 & 1 & 0.032 & 1.5 & 0.5 & 1 & - & & - & & 8 & 1 \\
\hline & $\Delta p s p F$ & - & & 0.19 & 1 & 0.032 & 1 & 0.016 & 0.75 & - & & 0.5 & 1 & - & & - & & 8 & 1 \\
\hline
\end{tabular}

AMP, ampicillin; CAZ, ceftazidime; FEP, cefepime; ETP, ertapenem; ATM, aztreonam; FOS, fosfomycin; IPM, imipenem; AK, amikacin; C, chloramphenicol.

${ }^{a} \mathrm{MIC}(\mathrm{mg} / \mathrm{L})$ of antimicrobial agent by gradient strip test.

${ }^{b}$ Fold reduction of MIC compared to the MIC of strains (wild-type SOS response)

'Wild-type (E. coli BW25113).

Cells with no data correspond to mutant-antimicrobial combinations that were not determined.

Green cells- susceptibility increased to antimicrobials; Yellow cells- No changes in susceptibility; Red cells- Resistance increased (relative to wild-type). 
$0.25 \mathrm{xMIC}$ relative to wild-type) of each antimicrobial agent. One hundred and fifty microliters of the diluted bacterial culture were then distributed among 96-well transparent flat bottom plates (Greiner Bio-One, Germany). Cultures were incubated at $37^{\circ} \mathrm{C}$ on an orbital shaker and agitated (2-mm orbital shaking, $450 \mathrm{rpm}, 10 \mathrm{~s}$ ) for $24 \mathrm{~h}$, and measured with an Infinite 200 PRO plate reader (Tecan). Optical density $\left(\mathrm{OD}_{595}\right)$ measurements were obtained every $20 \mathrm{~min}$. At least 4 biological replicates were measured for each condition in at least two independent assays.

\section{Time-Kill Curve Assays}

To show the effect of alteration of the stress response pathways on bacterial viability, time-kill curve assays were performed with the $\triangle p s p A, \triangle p s p B \quad \Delta p s p C$ mutants. MHB with $1 \mathrm{xMIC}$ concentrations of ceftazidime (CAZ), cefepime (FEP), ertapenem (ETP), ampicillin (AMP), and aztreonam (ATM) were used. Antimicrobial concentrations were relative to the MICs for strains harboring unmodified stress responses (wild-type). Growth in drug-free broth was evaluated in parallel as a control. Cultures were incubated at $37^{\circ} \mathrm{C}$ with shaking at $250 \mathrm{rpm}$. An initial inoculum of $10^{5} \mathrm{CFU} / \mathrm{mL}$ was used in all experiments; bacterial concentrations were determined at $0,2,4,6,8$, and $24 \mathrm{~h}$ by colony counting.

\section{Statistical Analysis}

All statistical analyses were performed using Graphpad Prism 6 software $^{1}$. The Student's $t$-test was used for statistical evaluation when two groups were compared. Differences were considered significant when $P<0.05$.

\section{RESULTS}

\section{Sensitization of $E$. coli to Antimicrobials Agents Determined by Disk Diffusion and Gradient Strip Test}

Twenty antimicrobials were tested by disk diffusion (Supplementary Table 3) in the initial screening (340 mutantdrug combinations were tested). Psp response was the most sensitized stress pathway with $22.5 \%$ of drug-gene deletion combinations affected, followed in descending order, by the Rcs (18\%), Bae (17.5\%), Cpx (13.7\%), and $\sigma^{\mathrm{E}}$ responses (2.5\%). To confirm these data, the gradient strip test (Table 1) was used to evaluate the activity of 9 antimicrobials (ampicillin, ceftazidime, cefepime, ertapenem, imipenem, aztreonam, amikacin, chloramphenicol, and fosfomycin) in 14 mutants $(\Delta r s e A, \Delta c p x A, \Delta c p x R, \Delta c p x P, \Delta r c s F, \Delta r c s C, \Delta r c s D, \Delta r c s B$, $\triangle$ baeR, $\triangle$ baes, $\triangle p s p A, \Delta p s p B, \Delta p s p C$, and $\Delta p s p F)$. The mutants that showed antimicrobial sensitization were the following: $\Delta r c s D$ showed a consistent 1.5- and 1.3-fold decrease in MIC values of aztreonam and fosfomycin, respectively; $\triangle b a e R$, showed a 1.5 -fold decline in the MIC of aztreonam; $\triangle p s p A$ showed 2-, 1. 4-, and 1.5-fold decreases in the MICs of ceftazidime (Supplementary Figure 1), cefepime and ertapenem,

${ }^{1}$ https://www.graphpad.com respectively; $\triangle p s p B$ showed a 2 - and 1.4 -fold decrease in the MIC of ceftazidime (Supplementary Figure 1) and cefepime, respectively, and finally, $\triangle p s p C$ showed a 1.5 -fold decrease in the MICs of ampicillin, ceftazidime and aztreonam.

Each mutant-antimicrobial combination that showed sensitization by gradient strips was tested with growth curves to analyze bacterial growth after short and long incubation periods in the presence of the antimicrobials cited above to confirm the previous results. The generalized sensitization of Psp mutants to beta-lactam agents (Table 1) led to growth curves even although no changes in MIC values were observed. The $\triangle p s p B$ mutant showed clear sensitization with differences for aztreonam, ceftazidime, cefepime and ertapenem relative to wild-type. After $24 \mathrm{~h}$, no growth was observed in the presence of aztreonam at $0.5 x$ MIC (optical density, OD value 0.32$)(p<0.01$, compared to wild-type, OD value 0.08) (Figures 1D-F), a decrease in OD was observed in the presence of ceftazidime at $0.25 x$ MIC (OD value 0.33$)(p<0.0001$, compared to wild-type BW25113, OD value 0.5) and at $0.5 x M I C$ (OD value 0.16 ) $(\mathrm{p}<0.05$, relative to wild-type, OD value 0.3$)$ (Figures 1M-O), and also in cefepime at $0.5 x M I C$ (OD value 0.11$)(p<0.05$, relative to wild-type, OD value 0.21) (Figures 1D-F). After $8 \mathrm{~h}$ of incubation, a decrease in OD was also observed at $0.25 \times$ MIC of cefepime (OD value 0.13 ) $(p<0.0001$, compared to wild-type, OD value 0.25 ) (Figures 1G-I) and at $0.5 x M I C$ of ertapenem (OD value 0.14$)(p<0.0001$, relative to wild-type, OD value $0.29)$ (Figures 1P-R). The $\triangle p s p A$ and $\triangle p s p C$ mutants showed no growth at $0.5 x M I C$ of aztreonam (OD value 0.10$)(p<0.01$, compared to wild-type, OD value 0.27 ) (Figures 1A-C) until $12 \mathrm{~h}$ and no growth was observed at $0.5 \mathrm{xMIC}$ of cefepime (OD value 0.08$)(p<0.0001$, relative to wild-type, OD value 0.30) (Figures $\mathbf{1 J - L}$ ) at $24 \mathrm{~h}$, respectively. No significant decrease in growth was observed for other mutant-antimicrobial combinations, and a paradoxical effect was observed with aztreonam (Supplementary Figures 2-4).

\section{The Impact of Psp Response Alteration on Bactericidal Activity of Beta-Lactam Antimicrobials}

Psp mutants $(\triangle p s p A, \Delta p s p B$, and $\Delta p s p C)$ were the selected mutants due to its significant sensitizing effect to antimicrobials compared to the wild-type and was therefore used for timekill assays to study cell viability in the presence of $1 \times$ MIC concentrations of beta-lactam agents selected. A bactericidal effect was observed for $\triangle p s p A$ and $\triangle p s p C$ mutants in the presence of AMP with drops up to $1 \log _{10}(p<0.01)$ at $8 \mathrm{~h}$ (Figures 2A,B). To note, a bacteriostatic effect was observed with the rest of the antimicrobials evaluated. At 1xMIC CAZ and ATM for $\triangle p s p A, \triangle p s p B$, and $\triangle p s p C$ mutants, reductions of $2.2 \log _{10}(p<0.0001), 0.9 \log _{10}(p=0.117, \mathrm{~ns})$ and 2.5 $\log _{10}(p<0.0001)$, respectively, were observed at $6-8 \mathrm{~h}$ for the first agent (Figures 2C-E), maintaining growth delay at $24 \mathrm{~h}$ and drops of $3.1 \log _{10}(p<0.01), 4 \log _{10}(p<0.01)$ and $3.9 \log _{10}(p<0.01)$, respectively, were observed at $4-$ $6 \mathrm{~h}$ for the second drug (Figures 2J-L). At 1xMIC ETP, reductions of $6 \log _{10}(p<0.01)$ for $\Delta p s p B$ mutant and $1.7 \log _{10}$ 


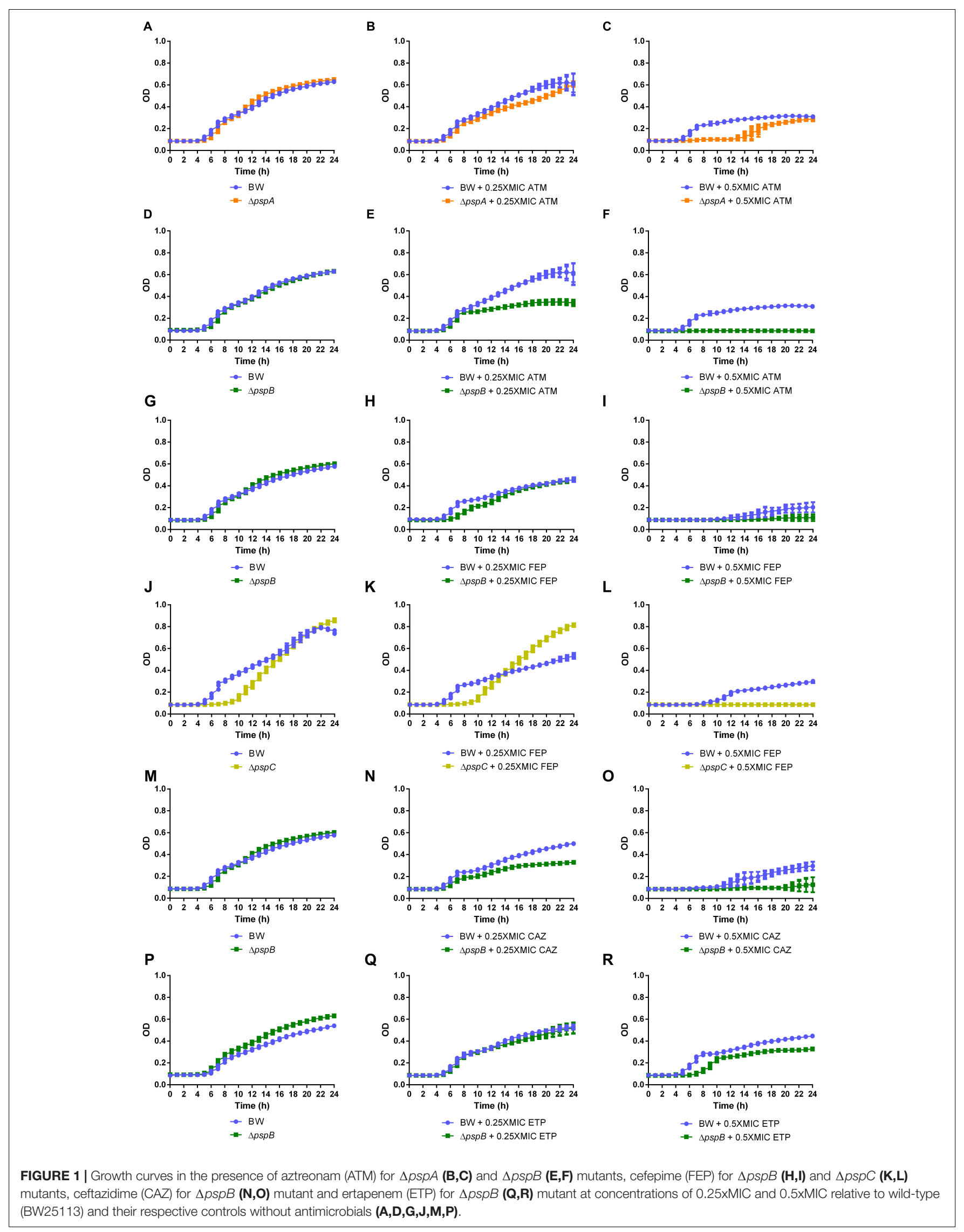




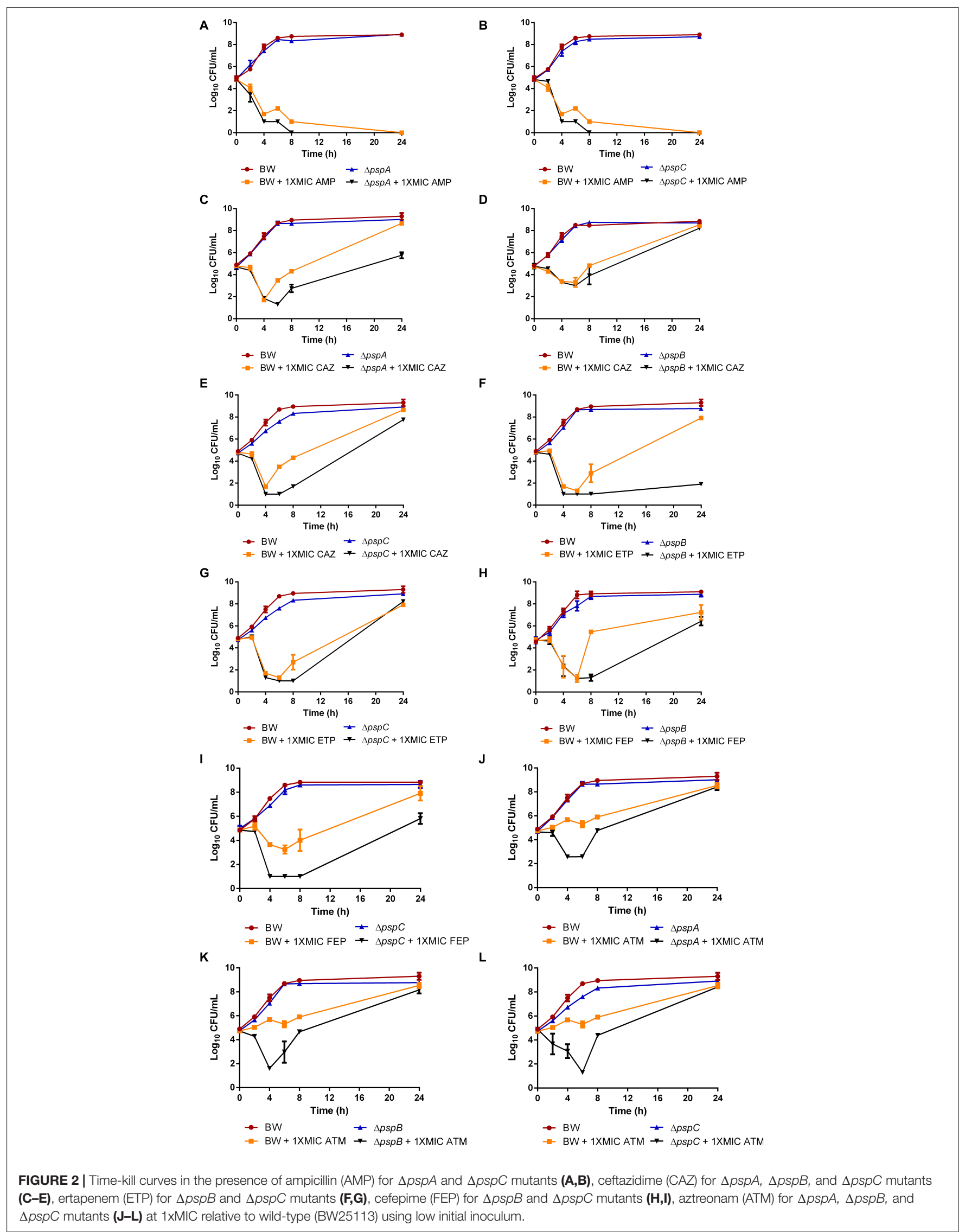


$(p<0.05)$ for $\triangle p s p C$ mutant were observed at 24 and $8 \mathrm{~h}$, respectively (Figures 2F,G) and drops of $4.2 \log _{10}(p<0.0001)$ for $\triangle p s p B$ mutant and $3 \log _{10}(\mathrm{p}<0.01)$ for $\Delta p s p C$ mutant were found after treatment with FEP at $8 \mathrm{~h}$ (Figures $2 \mathbf{H}, \mathbf{I}$ ). No differences in cell viability loss were observed for $\triangle p s p B$ mutant in the presence of ampicillin or for $\triangle p s p A$ mutant in the presence of ertapenem and cefepime (Supplementary Figures 5A-C).

\section{DISCUSSION}

Apart from the search for new drugs, new strategies are also necessary to prevent the emergence of resistance and extend the life of antimicrobial agents. Envelope stress responses are a set of coordinated physiological mechanisms that sense envelope damage or defects and trigger transcriptome alterations to mitigate this stress. In general terms, these pathways are focused on outer membrane stress ( $\sigma^{\mathrm{E}}$ response), inner membrane stress (Cpx, and Psp responses), damage through exposure to toxic molecules (Bae response) and alterations in outer membrane permeability, changes in peptidoglycan biosynthesis and defects in lipoprotein trafficking (Rcs response) (Mitchell and Silhavy, 2019). Psp-activated IM disruptions tend to be more severe than those required to activate $\mathrm{Cpx}$, being the first extensive disruptions that result in the loss of proton motive force (van der Laan et al., 2003; Maxson and Darwin, 2004; Becker et al., 2005), which could explain the greater effect on sensitization in strains deficient in this response.

Previous studies have evaluated the effect of mutations on the envelope stress response through deletion of certain genes (Nicoloff et al., 2017) or overactivation responses (McEwen and Silverman, 1980; Cosma et al., 1995; Danese et al., 1995), with sensitization to antimicrobials infrequently used in clinics (rifampicin or bacitracin) (Nicoloff et al., 2017) when RseA was deleted. In the present study, we evaluated a putative strategy consisting of disbalancing of envelope stress responses in the presence of antimicrobials from different families, including cell wall-disturbing agents (penicillins, cephalosporins, carbapenems, aztreonam, colistin, and fosfomycin), protein synthesis inhibitors (aminoglycosides, tetracyclines, and chloramphenicol), RNA synthesis inhibitors (rifampin), DNA synthesis inhibitors (fluoroquinolones) and folic acid synthesis inhibitors (sulfonamides and trimethoprim). It is important to highlight the clinical relevance of our set of selected antimicrobials for the treatment of infections caused by Gram-negative bacteria and the wide spectrum of targets covered, listed above. Gene deletions that affected sensitization to antimicrobials involved the Rcs response $(r c s D$, aztreonam and fosfomycin), the Bae response (baeR, aztreonam) and the Psp response ( $p s p A$, ceftazidime, cefepime and ertapenem; $p s p B$, ceftazidime and ertapenem; $p s p C$, ampicillin, ceftazidime, aztreonam). Beta-lactams constituted $83 \%$ of the antimicrobials to which strains were sensitized by gradient strip test, and the cell wall was the target in $100 \%$ of them.

Alteration of the Psp response was the envelope stress pathway with the greatest effect on sensitization in the presence of antimicrobials, as demonstrated by growth curves and timekill curve assays. Various components are involved in the Psp response, notably $\mathrm{PspB}$ (inner membrane protein). Under activating conditions, $\mathrm{PspB}$ and $\mathrm{PspC}$ interact with PspA (PspF inhibitor), which releases $\mathrm{PspF}$ (response regulator) (Yamaguchi et al., 2013), which interacts with RNA polymerase to increase psp gene transcription (Jovanovic et al., 1996; Lloyd et al., 2004). Specifically, deletion of the $p s p A, p s p B$ and $p s p C$ genes had the greatest impact on cell viability and bacterial growth in the presence of beta-lactams antimicrobials, mainly ampicillin, aztreonam, cefepime, ceftazidime and ertapenem, enhancing the bactericidal effect of this family of agents.

Another important aspect is that the target of these antimicrobials in the cell wall of $E$. coli is primarily PBP3 (ampicillin, cefepime, ceftazdime, aztreonam) and PBP2 (ertapenem) which are involved in cell division whose inhibition lead to filamentation and the formation of spherical cells, respectively (Hayes and Orr, 1983; Bush and Bradford, 2016; Rodvold et al., 2018). Nevertheless, the underlying interaction between these agents and the Psp response proteins is unknown. We could hypothesize that the double damage of the bacterial envelope: inner membrane damage due to $p s p$ deletion and the beta-lactam antimicrobials effect acting on the PBPs proteins, trigger a sensitization effect reducing bacterial growth a increasing antimicrobial lethality.

In general terms, the effect on antimicrobial sensitization in the tested mutants was moderate but consistent; however, it could be interesting to test other essential genes ( $r p o E$, degS, rseP, cpxQ, $i g a A$ ) involved in envelope stress responses, although these were not available in the KEIO collection.

The emergence of innovative therapeutic strategies, in combination with more conventional approaches, is advancing our understanding of interactions between microbiota, host and pathogenic bacteria. Questions that remain to be answered include how disruption of the envelope stress response could impact not only harmful bacteria, but also healthy ones, causing microbiota impairment and associated disorders, such as C. difficile infection (Bäumler and Sperandio, 2016).

In conclusion, a defective Psp envelope stress response increases E. coli susceptibility to beta-lactams antimicrobials, and is particularly remarkable with aztreonam, cefepime, ceftazidime and ertapenem. The role of this system in repairing extensive disruptions to the inner membrane makes this pathway essential to bacterial survival. Its use as a potential target for bacterial sensitization deserves in-depth evaluation.

\section{DATA AVAILABILITY STATEMENT}

The raw data supporting the conclusions of this article will be made available by the authors, without undue reservation.

\section{AUTHOR CONTRIBUTIONS}

VF, SD-D, and AG-D performed the lab assays. ER interpreted the data and wrote the manuscript with input from all coauthors. JR-M and ER designed and guided the execution 
of all experiments. FD-P, ÁP, and JR-M supervised the project and contributed to the interpretation of the results and valuable discussion. All authors contributed to the article and approved the submitted version.

\section{FUNDING}

This study was supported by the Instituto de Salud Carlos III, Ministerio de Economía y Competitividad-co-financed by European Development Regional Fund "A way to achieve Europe" ERDF, Spanish Network for Research in Infectious Diseases [REIPI RD12/0015 and RD16/0016]. Supported by the Plan Nacional de I+D+i 2013-2016 and Instituto de

\section{REFERENCES}

Ades, S. E. (2004). Control of the alternative sigma factor $\sigma \mathrm{E}$ in Escherichia coli. Curr. Opin. Microbiol. 7, 157-162. doi: 10.1016/j.mib.2004.02.010

Baba, T., Ara, T., Hasegawa, M., Takai, Y., Okumura, Y., Baba, M., et al. (2006). Construction of Escherichia coli K-12 in-frame, single-gene knockout mutants: the Keio collection. Mol. Syst. Biol. 2:msb4100050. doi: 10.1038/msb410 0050

Baranova, N., and Nikaido, H. (2002). The BaeSR two-component regulatory system activates transcription of the yegMNOB (mdtABCD) transporter gene cluster in Escherichia coli and increases its resistance to novobiocin and deoxycholate. J. Bacteriol. 184, 4168-4176. doi: 10.1128/JB.184.15.4168-4176. 2002

Bäumler, A. J., and Sperandio, V. (2016). Interactions between the microbiota and pathogenic bacteria in the gut. Nature 535, 85-93. doi: 10.1038/ nature 18849

Becker, L. A., Bang, I.-S., Crouch, M.-L., and Fang, F. C. (2005). Compensatory role of PspA, a member of the phage shock protein operon, in rpoE mutant Salmonella enterica serovar Typhimurium. Mol. Microbiol. 56, 1004-1016. doi: 10.1111/j.1365-2958.2005.04604.x

Bush, K., and Bradford, P. A. (2016). $\beta$-Lactams and $\beta$-Lactamase inhibitors: an overview. Cold Spring Harb. Perspect. Med. 6:a025247. doi: 10.1101/cshperspect. a025247

Cattoir, V., and Felden, B. (2019). Future antibacterial strategies: from basic concepts to clinical challenges. J. Infect. Dis. 220, 350-360. doi: 10.1093/infdis/ jiz134

Clinical and Laboratory Standards Institute (2016). Performance Standards for Antimicrobial Susceptibility Testing: Twenty-Sixth Informational Supplement M100-S26. Wayne, PA: CLSI.

Cordeiro, R. P., Krause, D. O., Doria, J. H., and Holley, R. A. (2014). Role of the BaeSR two-component regulatory system in resistance of Escherichia coli O157:H7 to allyl isothiocyanate. Food Microbiol. 42, 136-141. doi: 10.1016/j.fm. 2014.03.011

Cosma, C. L., Danese, P. N., Carlson, J. H., Silhavy, T. J., and Snyder, W. B. (1995). Mutational activation of the Cpx signal transduction pathway of Escherichia coli suppresses the toxicity conferred by certain envelope-associated stresses. Mol. Microbiol. 18, 491-505. doi: 10.1111/j.1365-2958.1995.mmi_1803 0491.X

Danese, P. N., Snyder, W. B., Cosma, C. L., Davis, L. J., and Silhavy, T. J. (1995). The Cpx two-component signal transduction pathway of Escherichia coli regulates transcription of the gene specifying the stress-inducible periplasmic protease, DegP. Genes Dev. 9, 387-398. doi: 10.1101/gad.9.4.387

DIckey, S. W., Cheung, G. Y. C., and Otto, M. (2017). Different drugs for bad bugs: antivirulence strategies in the age of antibiotic resistance. Nat. Rev. Drug Discov. 16, 457-471. doi: 10.1038/nrd.2017.23

Erickson, K. D., and Detweiler, C. S. (2006). The Rcs phosphorelay system is specific to enteric pathogens/commensals and activates ydeI, a gene important for persistent Salmonella infection of mice. Mol. Microbiol. 62, 883-894. doi: 10.1111/j.1365-2958.2006.05420.x
Salud Carlos III, Subdirección General de Redes y Centros de Investigación Cooperativa, Ministerio de Economía, Industria y Competitividad, Spanish Network for Research in Infectious Diseases (PI14/00940, PI17/01501, PI20/00239, RD16/0016/0001, and REIPI RD16/0016/0009)-co-financed by European Development Regional Fund "A way to achieve Europe," Operative Programme Intelligent Growth 2014-2020.

\section{SUPPLEMENTARY MATERIAL}

The Supplementary Material for this article can be found online at: https://www.frontiersin.org/articles/10.3389/fmicb. 2021.653479/full\#supplementary-material

Farizano, J. V., Torres, M. A., Pescaretti, M. L. M., and Delgado, M. A. (2014). The RcsCDB regulatory system plays a crucial role in the protection of Salmonella enterica serovar Typhimurium against oxidative stress. Microbiology 160, 21902199. doi: 10.1099/mic.0.081133-0

Farris, C., Sanowar, S., Bader, M. W., Pfuetzner, R., and Miller, S. I. (2010). Antimicrobial peptides activate the rcs regulon through the outer membrane lipoprotein RcsF. J. Bacteriol. 192, 4894-4903. doi: 10.1128/JB.00505-10

Flores-Kim, J., and Darwin, A. J. (2014). Regulation of bacterial virulence gene expression by cell envelope stress responses. Virulence 5, 835-851. doi: 10.4161/ 21505594.2014.965580

Flores-Kim, J., and Darwin, A. J. (2016). The phage shock protein response. Annu. Rev. Microbiol. 70, 83-101. doi: 10.1146/annurev-micro-102215-095359

Guest, R. L., and Raivio, T. L. (2016). Role of the gram-negative envelope stress response in the presence of antimicrobial agents. Trends Microbiol. 24, 377-390. doi: 10.1016/j.tim.2016.03.001

Hayes, M. V., and Orr, D. C. (1983). Mode of action of ceftazidime: affinity for the penicillin-binding proteins of Escherichia coli K12, Pseudomonas aeruginosa and Staphylococcus aureus. J. Antimicrob. Chemother. 12, 119-126. doi: 10.1093/ jac/12.2.119

Hersch, S. J., Watanabe, N., Stietz, M. S., Manera, K., Kamal, F., Burkinshaw, B., et al. (2020). Envelope stress responses defend against type six secretion system attacks independently of immunity proteins. Nat. Microbiol. 5, 706-714. doi: 10.1038/s41564-020-0672-6

Hirakawa, H., Nishino, K., Hirata, T., and Yamaguchi, A. (2003). Comprehensive studies of drug resistance mediated by overexpression of response regulators of two-component signal transduction systems in Escherichia coli. J. Bacteriol. 185, 1851-1856. doi: 10.1128/JB.185.6.1851-1856.2003

Huang, Y.-H., Ferrières, L., and Clarke, D. J. (2006). The role of the Rcs phosphorelay in Enterobacteriaceae. Res. Microbiol. 157, 206-212. doi: 10.1016/ j.resmic.2005.11.005

Jovanovic, G., Weiner, L., and Model, P. (1996). Identification, nucleotide sequence, and characterization of $\mathrm{PspF}$, the transcriptional activator of the Escherichia coli stress-induced psp operon. J. Bacteriol. 178, 1936-1945. doi: 10.1128/JB.178.7.1936-1945.1996

Kim, D. Y. (2015). Two stress sensor proteins for the expression of sigmaE regulon: DegS and RseB. J. Microbiol. 53, 306-310. doi: 10.1007/s12275-015-5112-6

Laubacher, M. E., and Ades, S. E. (2008). The Rcs phosphorelay is a cell envelope stress response activated by peptidoglycan stress and contributes to intrinsic antibiotic resistance. J. Bacteriol. 190, 2065-2074. doi: 10.1128/JB.01740-07

Laxminarayan, R., Duse, A., Wattal, C., Zaidi, A. K. M., Wertheim, H. F. L., Sumpradit, N., et al. (2013). Antibiotic resistance-the need for global solutions. Lancet Infect. Dis. 13, 1057-1098. doi: 10.1016/S1473-3099(13)70318-9

Lima, S., Guo, M. S., Chaba, R., Gross, C. A., and Sauer, R. T. (2013). Dual Molecular Signals mediate the bacterial response to outer-membrane stress. Science 340, 837-841. doi: 10.1126/science.1235358

Lin, M.-F., Lin, Y.-Y., Yeh, H.-W., and Lan, C.-Y. (2014). Role of the BaeSR two-component system in the regulation of Acinetobacter baumannii adeAB genes and its correlation with tigecycline susceptibility. BMC Microbiol. 14:119. doi: 10.1186/1471-2180-14-119 
Lloyd, L. J., Jones, S. E., Jovanovic, G., Gyaneshwar, P., Rolfe, M. D., Thompson, A., et al. (2004). Identification of a new member of the phage shock protein response in Escherichia coli, the phage shock protein G (PspG). J. Biol. Chem. 279, 55707-55714. doi: 10.1074/jbc.M408994200

Majdalani, N., and Gottesman, S. (2005). The Rcs phosphorelay: a complex signal transduction system. Annu. Rev. Microbiol. 59, 379-405. doi: 10.1146/annurev. micro.59.050405.101230

Maxson, M. E., and Darwin, A. J. (2004). Identification of inducers of the Yersinia enterocolitica phage shock protein system and comparison to the regulation of the RpoE and Cpx extracytoplasmic stress responses. J. Bacteriol. 186, 4199-4208. doi: 10.1128/JB.186.13.4199-4208.2004

McEwen, J., and Silverman, P. (1980). Chromosomal mutations of Escherichia coli that alter expression of conjugative plasmid functions. Proc. Natl. Acad. Sci.U.S.A. 77, 513-517. doi: 10.1073/pnas.77.1.513

Mitchell, A. M., and Silhavy, T. J. (2019). Envelope stress responses: balancing damage repair and toxicity. Nat. Rev. Microbiol. 17, 417-428. doi: 10.1038/ s41579-019-0199-0

Nicoloff, H., Gopalkrishnan, S., and Ades, S. E. (2017). Appropriate regulation of the $\sigma \mathrm{E}$-dependent envelope stress response is necessary to maintain cell envelope integrity and stationary-phase survival in Escherichia coli. J. Bacteriol. 199:e00089-17. doi: 10.1128/JB.00089-17

Raffa, R. G., and Raivio, T. L. (2002). A third envelope stress signal transduction pathway in Escherichia coli. Mol. Microbiol. 45, 1599-1611. doi: 10.1046/j.13652958.2002.03112.x

Raivio, T. L. (2014). Everything old is new again: an update on current research on the Cpx envelope stress response. Biochim. Biophys. Acta Mol. Cell Res. 1843, 1529-1541. doi: 10.1016/j.bbamcr.2013.10.018

Recacha, E., Machuca, J., Díaz de Alba, P., Ramos-Güelfo, M., Docobo-Pérez, F., Rodriguez-Beltrán, J., et al. (2017). Quinolone resistance reversion by targeting the SOS response. MBio 8:e00971-17. doi: 10.1128/mBio.00971-17

Rodvold, K. A., Gotfried, M. H., Chugh, R., Gupta, M., Patel, A., Chavan, R., et al. (2018). Plasma and intrapulmonary concentrations of cefepime and zidebactam following intravenous administration of WCK 5222 to healthy adult subjects. Antimicrob. Agents Chemother. 62:e00682-18. doi: 10.1128/AAC.00 682-18

Ruiz, N., and Silhavy, T. J. (2005). Sensing external stress: watchdogs of the Escherichia coli cell envelope. Curr. Opin. Microbiol. 8, 122-126. doi: 10.1016/j. mib.2005.02.013

Silhavy, T. J., Kahne, D., and Walker, S. (2010). The bacterial cell envelope. Cold Spring Harb. Perspect. Biol. 2:a000414. doi: 10.1101/cshperspect.a000414

Valentin-Hansen, P., Johansen, J., and Rasmussen, A. A. (2007). Small RNAs controlling outer membrane porins. Curr. Opin. Microbiol. 10, 152-155. doi: 10.1016/j.mib.2007.03.001

van der Laan, M., Urbanus, M. L., ten Hagen-Jongman, C. M., Nouwen, N., Oudega, B., Harms, N., et al. (2003). A conserved function of YidC in the biogenesis of respiratory chain complexes. Proc. Natl. Acad. Sci. U.S.A. 100, 5801-5806. doi: 10.1073/pnas.0636761100

Yamaguchi, S., Reid, D. A., Rothenberg, E., and Darwin, A. J. (2013). Changes in Psp protein binding partners, localization and behaviour upon activation of the Yersinia enterocolitica phage shock protein response. Mol. Microbiol. 87, 656-671. doi: $10.1111 / \mathrm{mmi} .12122$

Conflict of Interest: The authors declare that the research was conducted in the absence of any commercial or financial relationships that could be construed as a potential conflict of interest.

Copyright ๑ 2021 Recacha, Fox, Díaz-Díaz, García-Duque, Docobo-Pérez, Pascual and Rodríguez-Martínez. This is an open-access article distributed under the terms of the Creative Commons Attribution License (CC BY). The use, distribution or reproduction in other forums is permitted, provided the original author(s) and the copyright owner(s) are credited and that the original publication in this journal is cited, in accordance with accepted academic practice. No use, distribution or reproduction is permitted which does not comply with these terms. 